\title{
The influence of microstructure characteristics on electrical properties in ITO thin film
}

\author{
Chenggang Yang ${ }^{1}$, Jingyuan Yang ${ }^{1, *}$, Dongao Han ${ }^{1}$, Chan $\mathrm{Li}^{1}$,Yuting Xu ${ }^{1}$, and Yang Qiu ${ }^{2}$ \\ ${ }^{1}$ Nuclear and Radiation Safety Centre MEE, Beijing 102400, China \\ ${ }^{2}$ NICE Solar Energy Corporation Ltd., Beijing 102211, China
}

\begin{abstract}
ITO thin films were deposited on sapphire substrates under different deposition parameters via ion-assistant electron beam evaporation method. Microstructure characteristics such as crystalline structure and surface morphology of as-deposited ITO thin films were studied by using X-ray diffraction spectroscopy, transmission electron microscopy and field emission scanning electron microscopy, as well as the index of sheet resistance, carrier concentration, carrier mobility and transmission in visible spectrum were tested by means of Hall effect tester and UV-VIS-NIR spectrophotometer, respectively. The influence and impact mechanism of microstructure characteristic on electrical properties of as-deposited ITO thin films were investigated and analyzed in detail.
\end{abstract}

\section{Preface}

Sn doped $\operatorname{In}_{2} \mathrm{O}_{3}$ (ITO) ${ }^{[1]}$ is a kind of semiconductor oxide thin film ${ }^{[2,3]}$. Due to the excellent combination of wide band gap $\left(>3.5 \mathrm{eV}^{[4]}\right)$, low resistivity, high transmittance in visible spectrum $(>95 \%$ at $550 \mathrm{~nm})$, high IR reflectance $(>80 \%)$ and other unique physical properties $^{[5]}$, ITO thin films were widely used in varieties of industries such as flat panel displays, LEDs, solar panels, thermal mirrors, electrochromic devices ${ }^{[6,7]}$, etc.

In all of the applications, ITO thin films are required to present excellent electric and optical properties. For this reason, researchers have spent great efforts on investigating ITO's electrical and optical properties during last several decades. However, due to the coating mechanisms of different deposition methods and the complex effects of various process parameters involved in, the electrical and optical properties of ITO thin films often conducted diversely or even inversely to the deposition parameters according to the past research works. As a result, there is still controversy in the understanding to the mechanisms of electrical and optical properties of ITO thin films. Therefore, further studies on the electrical and optical properties of ITO thin films still show great significance. The theoretical calculation shows that the carrier mobility of $\operatorname{In}_{2} \mathrm{O}_{3}$ single crystal can be as high as $160 \mathrm{~cm}^{2} \mathrm{~V}^{-1} \mathrm{~s}^{-1[8]}$, therefore, the resistivity of ITO thin films can be as low as $4.4 \times 10^{-6}$ $\Omega \cdot \mathrm{cm}$ theoretically. Practically, Suzuki et al. ${ }^{[9]}$ obtained high-quality ITO thin film via PLD method at $300^{\circ} \mathrm{C}$, the transmittance in visible spectrum was over $90 \%$, the resistivity was as low as $7.2 \times 10^{-5} \Omega \cdot \mathrm{cm}$, and the carrier mobility was as high as $2.5 \times 1021 \mathrm{~cm}^{-3}$; Ohta et al ${ }^{[10]}$ studied ITO thin film with ultra-flatness and intense (400) texture which was epitaxial grown on single crystal YSZ (Yttria-Stabilized Zirconia) substrate at $600^{\circ} \mathrm{C}$ via PLD, and its resistivity was as low as $7.7 \times 10^{-5} \Omega \cdot \mathrm{cm}$, the carrier mobility was as high as $1.9 \times 1021 \mathrm{~cm}^{-3}$, and the Hall mobility was $44 \mathrm{~cm}^{2} \mathrm{~V}^{-1} \mathrm{~s}^{-1}$, which was the most remarkable electrical property obtained by conventional methods reported in the literature. It can be seen that there is still a large gap between the current electrical properties of ITO thin films and theoretical calculation, which indicates that more profound researches are still indispensable. Therefore, studies on precise control of the electrical and optical properties of ITO thin films are particularly significant.

In this paper, ITO thin films were deposited on sapphire substrates under different deposition parameters via ion-assistant electron beam evaporation method. Microstructure characteristics such as crystalline structure and surface morphology of as-deposited ITO thin films were studied by using X-ray diffraction spectroscopy, transmission electron microscopy and field emission scanning electron microscopy, as well as the index of sheet resistance, carrier concentration, carrier mobility and transmission in visible spectrum were tested by means of Hall effect tester and UV-VIS-NIR spectrophotometer, respectively. The influence and impact mechanism of microstructure characteristic on electrical properties of as-deposited ITO thin films were investigated and analyzed in detail.

\section{Experiment}

* Corresponding author: yjyle@163.com 


\subsection{Sample preparation}

ITO thin films were deposited by ion-assisted electron beam evaporation (EB). During the deposition process, $\mathrm{O}_{2}$ with high purity was introduced into the cathode of Hall ion source and was ionized by high-speed electron bombardment, the $\mathrm{O}$ - beam formed was utilized to ion cleaning and ion assistance during the deposition. The $\mathrm{O}_{2}$ flow was regulated by the mass flow control meter (MFC). Sapphire $(\Phi=15 \mathrm{~mm}, \mathrm{~d}=2 \mathrm{~mm})$ was used as substrate, and ultrasonically cleaned with acetone, alcohol and deionized water in sequence, and then fixed on the fixture after drying. Before deposition, the chamber was evacuated to a background vacuum of 9.0 $\times 10-5 \mathrm{~Pa}$, the substrates were heated to $300^{\circ} \mathrm{C}$ and baked for 30 minutes. ITO particles with high purity were used as the evaporation material $\left(\mathrm{In}_{2} \mathrm{O}_{3}: \mathrm{SnO}_{2}=9: 1 \%, \mathrm{D}=1 \sim 4\right.$ $\mathrm{mm}$, Umicore). $270^{\circ}$ E-type electron gun was used as the thermal source, the deposition rate was adjusted by regulating the current of electron beam. The film thickness and deposition rate were monitored by the quartz crystal microbalance (QCM) in real-time. ITO thin films were deposited under different deposition parameters. First, the oxygen flow rate was fixed at $45 \mathrm{sccm}$, and the ITO thin films were deposited at deposition rates of $1 \AA / \mathrm{s}, 2 \AA / \mathrm{s}, 3 \AA / \mathrm{s}$, and $4 \AA / \mathrm{s}$. Samples were marked as: R1 R4; Secondly, the deposition rate was fixed at $2 \AA / \mathrm{s}$, and the depositions were conducted under oxygen flow rates of $30,40,50$ and $60 \mathrm{sccm}$, respectively. Samples were marked as: $\mathrm{O}_{30} \sim \mathrm{O}_{60}$. The thickness of the films was set to $250 \mathrm{~nm}$, and no post annealing treatment was implemented afterwards.

\subsection{Characterization}

The microstructure characteristics of ITO thin films were characterized by X-ray diffractometer (XRD, Bruker D8 advance). The test conditions were as follows: $\mathrm{Cu}$ target $\mathrm{K} \alpha$ radiation, $\lambda=0.15406 \mathrm{~nm}, \theta-2 \theta$ scanning method, scanning range was $10^{\circ} \sim 80^{\circ}$. The transmission electron microscope bright-field image and selected area electron diffraction (SAED) spectrum of thecross-section were tested by FEI Tecnai T20 transmission electron microscope. The morphology was taken by Hitachi S4800 field emission scanning electron microscope (FESEM). The sheet resistance, resistivity, carrier concentration and carrier mobility at room temperature were tested by means of Van der Pauw method via HL5500PC Hall tester (Nanometrics Inc.). Voltage: 20 $\mathrm{mV}$; DC: $1 \mathrm{~mA}$; magnetic field strength: $0.53 \mathrm{~T}$. The transmittance of ITO thin film in the visible spectrum was measured by Shimadzu UV-3600 UV-VIS-NIR spectrophotometer with a resolution of $1 \mathrm{~nm}$.

\section{Results and discussions}

\subsection{Structural characteristics}

XRD pattern of the ITO thin films obtained is shown in Figure 1. The comparison with the standard PDF card JCPDS 06-0416 shows that ITO thin film as-deposited are polycrystalline, with body-centered cubic structure (bixbyite, spatial point group: Ia-3 No.206) and (222) textured. The film grain size (see Table 1) D is estimated by Scherrer formula ${ }^{[11]}$.

$$
D=\frac{0.9 \lambda}{\beta \cdot \cos \theta}
$$

where, $\lambda$ is the wavelength of the $\mathrm{X}$-ray used for testing, $\beta$ is the FWHM (full width at half maximum) of the diffraction peak corresponding to a certain $2 \theta$ angle. The calculation result shows that the average grain size of the ITO thin films increases with the increasing of deposition rate, while the variation of oxygen flow rate shows no obvious influence on the grain size.

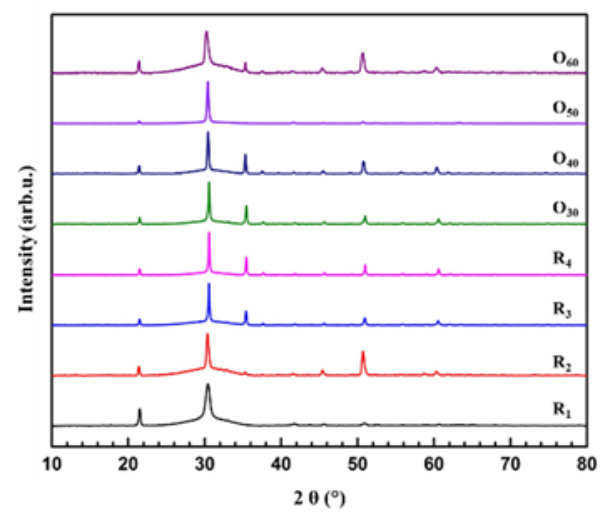

Fig.1 XRD pattern for ITO thin films deposited at different parameters.

Table 1. Physical properties of as-deposited ITO thin films

\begin{tabular}{|c|c|c|c|c|c|c|c|c|}
\hline & $\mathbf{R}_{\mathbf{1}}$ & $\mathbf{R}_{\mathbf{2}}$ & $\mathbf{R}_{\mathbf{3}}$ & $\mathbf{R}_{\mathbf{4}}$ & $\mathbf{O}_{\mathbf{3 0}}$ & $\mathbf{O}_{\mathbf{4 0}}$ & $\mathbf{O}_{\mathbf{5 0}}$ & $\mathbf{O}_{\mathbf{6 0}}$ \\
\hline Grain size $(\mathrm{nm})$ & 65.2 & 70.4 & 85.8 & 110.7 & 74.0 & 82.7 & 72.9 & 124.1 \\
\hline Sheet resistance $(\Omega / \square)$ & 28.5 & 11.9 & 18.3 & 21.7 & 21.7 & 27.1 & 10.3 & 10.1 \\
\hline Resistivity $\left(10^{-4} \Omega \cdot \mathrm{cm}\right)$ & 7.14 & 2.99 & 4.57 & 5.42 & 5.43 & 6.77 & 2.58 & 2.53 \\
\hline Electron mean free path $(\mathrm{nm})$ & 11.8 & 3.9 & 3.8 & 4.1 & 5.2 & 7.5 & 3.3 & 2.9 \\
\hline
\end{tabular}

It can also be observed from Figure 1 that the diffraction peak intensity of the (400) crystal plane of the ITO thin films gradually increases with the increasing of deposition rate, and decreases with the increasing of oxygen flow rate within the range of $30-50 \mathrm{sccm}$, but rises slightly at oxygen flow rate of $60 \mathrm{sccm}$. The enhancement of the texture on (400) plane is usually caused by the increasing in the concentration of oxygen vacancies. In the experiment, with the increasing of the growth rate or the decreasing of the oxygen flow rate, 
the relative oxygen content along the growth surface of the film gradually decreased, resulting in an increase in the growth trend along (400) crystal plane in the film; On the other hand, when the oxygen flow rate reached $60 \mathrm{sccm}$, The oxygen flow per unit time exceeded the upper limit for the ionization capability of the Hall source. The surplus oxygen atoms led pressure in the vacuum chamber to rise, which reduced the mean free path of oxygen ions, prevent the oxygen ions from reacting with the growing film completely, and increased the oxygen vacancy concentration, led the film to grow along the (400) plane to a certain extent.

In addition, the XRD peaks of sample $R_{1}$ and $R_{2}$ both show a certain degree of broadening. D. Mergel et al. ${ }^{[1]}$ believes that this is caused by the segregation of neutral $\mathrm{SnO}_{2}$ at the grain boundaries of the film in the oxygenrich environment. In the experiment, $\mathrm{R}_{1}$ was selected as the sample of TEM test. The SAED spectrum of the cross-section is shown in Figure 2(a). The inset is the TEM brightfield image of the selected area. We can see that in addition to the (211), (222), (400), (440) and (622) crystal plane diffraction rings of cubic $\mathrm{In}_{2} \mathrm{O}_{3}$, dim diffraction spots can also be observed at the position indicated by the arrow, with d values of $3.31 \AA, 3.34 \AA$, and $3.32 \AA$ (Figure 2 (b), (c), (d)). Among the possible composite, only the (110) crystal plane $(d=3.3470 \AA$, JCPDS 41-1445) of tetragonal $\mathrm{SnO}_{2}$ has corresponding interplanar spacing value. This indicates that there are $\mathrm{SnO}_{2}$ crystallites in the film, but due to the relatively low content, no obvious diffraction peaks can be observed in
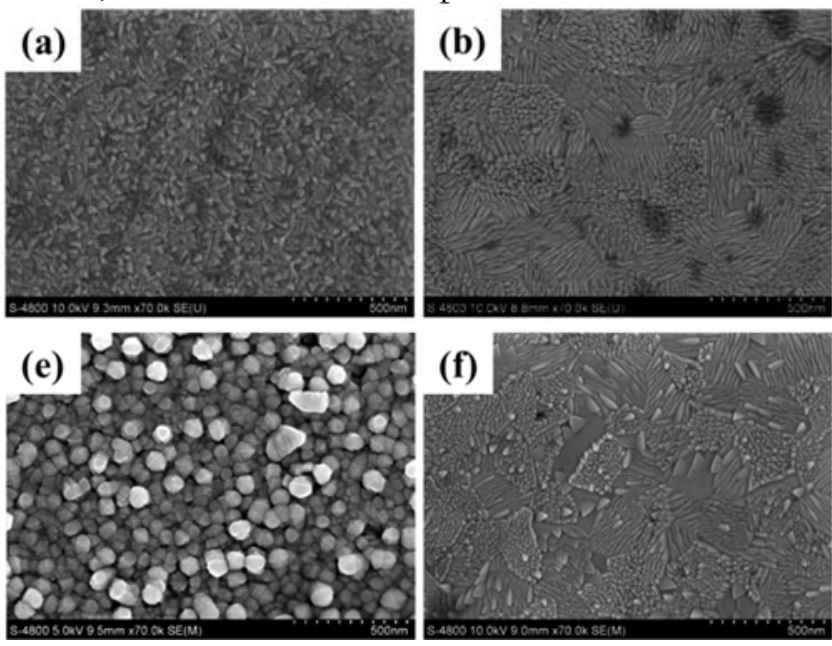

Fig.3 SEM images of ITO thin films deposited at different parameters.

$$
\rho=\frac{1}{\sigma}=\frac{1}{N e \mu}
$$

\subsection{Electrical Performance}

The sheet resistance and resistivity of the ITO film tested by the Van der Pauw method are listed in Table 1. For semiconductor transparent conductive oxide film materials, the resistivity $\rho$ and conductivity $\sigma$ are in reverse proportion to each other: the XRD pattern. The possible reason for its formation is that when the deposition rate is low, the growth surface of the film is in a relatively oxygen-rich environment, and a fraction of $\mathrm{Sn}$ ions are oxidized and segregate at the grain boundaries by the form of neutral $\mathrm{SnO}_{2}$.

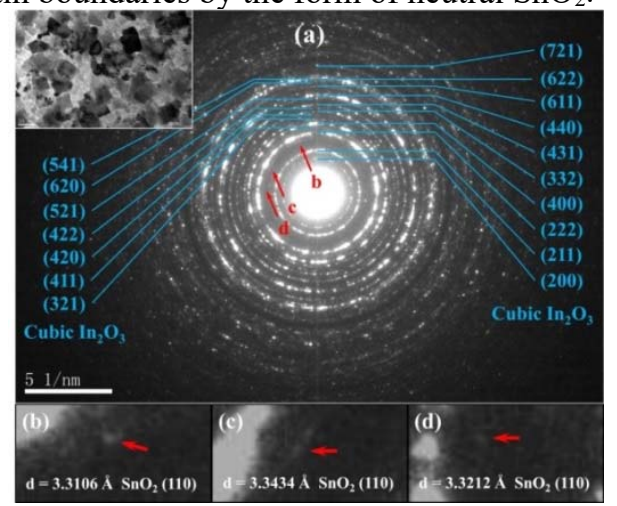

Fig.2 SAED image of ITO thin film. Inset at top left corner shows corresponding bright field image of ITO thin film, appended images (b), (c), (d) show the diffraction spots of $\mathrm{SnO} 2(110)$.

Figure 3 shows SEM morphology photos of ITO thin films prepared under different parameters. We can see that the grain size of the ITO thin film increases with the increasing of deposition rate, which is consistent with the calculation results in the above XRD analysis. With the increasing of the deposition rate, the surface of the film becomes rougher and structural defects increase.
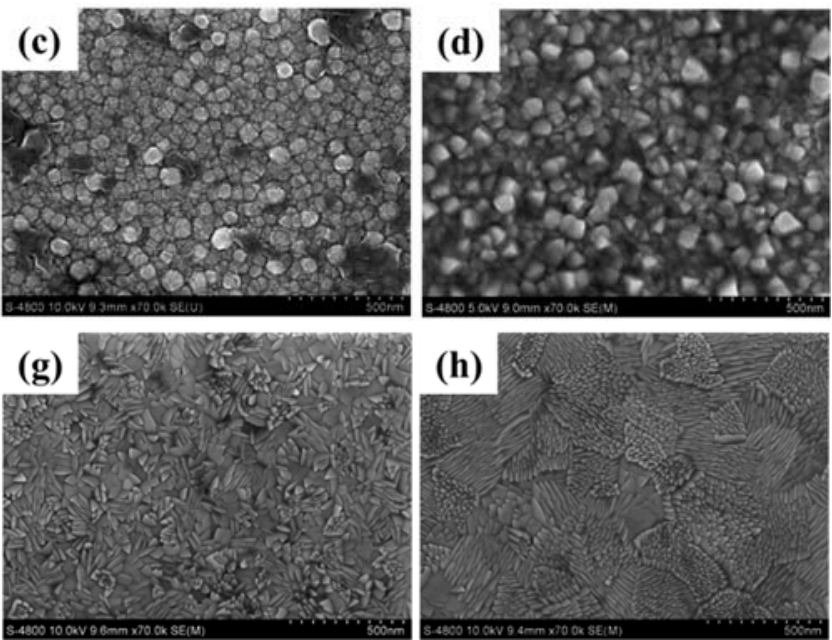
respectively. As can be seen from the figure, as the deposition rate increased, the carrier concentration of the ITO film gradually increased, Samples $\mathrm{R}_{1} \sim \mathrm{R}_{4}$ are $3.38 \times$ $1020 \mathrm{~cm}^{-3}, 4.82 \times 1020 \mathrm{~cm}^{-3}, 9.17 \times 1020 \mathrm{~cm}^{-3}$ and 1.1 $\times 1021 \mathrm{~cm}^{-3}$ respectively. The carrier concentration

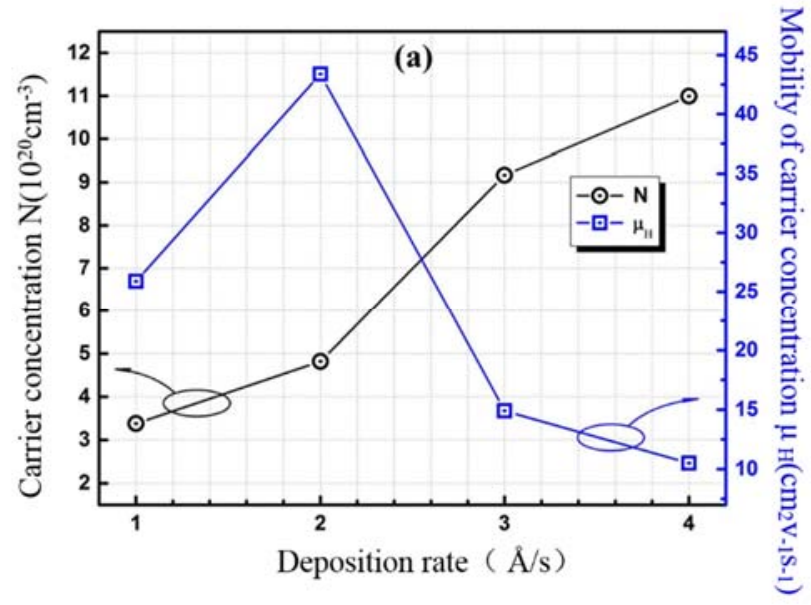

decreased initially and increased afterwards with the increasing of oxygen flow rate, $\mathrm{O}_{30} \sim \mathrm{O}_{50}$ are $7.60 \times 1020$ $\mathrm{cm}^{-3}, \quad 6.11 \times 1020 \mathrm{~cm}^{-3}, \quad 5.06 \times 1020 \mathrm{~cm}^{-3}$ and $\mathrm{O}_{60}$ is $5.80 \times 1020 \mathrm{~cm}^{-3}$ respectively.

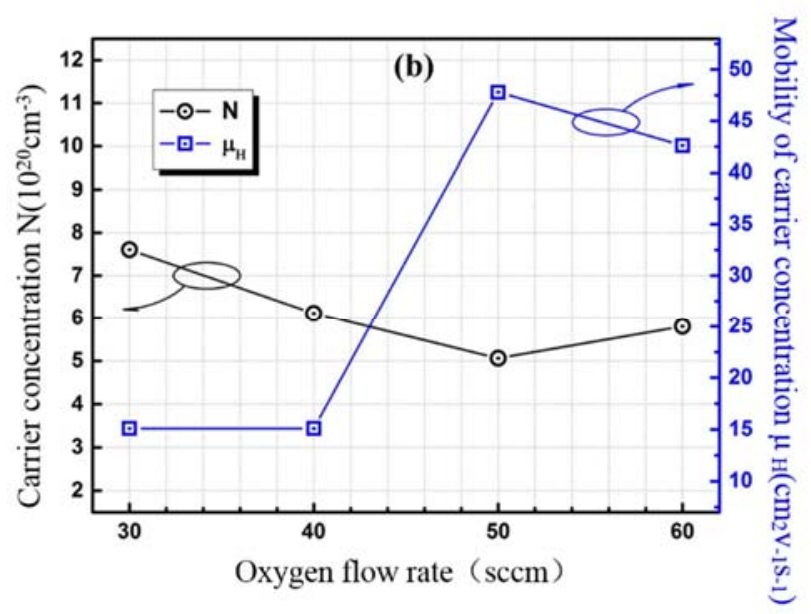

Fig.4 Electrical properties of as-deposited ITO thin films

In ITO thin film, the main sources of carriers are intrinsic oxygen vacancies and the substitutional doping of $\mathrm{Sn}^{4+}$ toIn ${ }^{3+}$. XRD test results show that the increase in the deposition rate enhances (400) texture in the film, which indicates that the oxygen vacancy concentration in the film increases with the increasing of deposition rate. The film grows in a relatively oxygen-rich environment at a low deposition rate, the evaporated particles are fully oxidized making the oxygen vacancy concentration small in the film, the relatively oxygen-deficient environment formed during the growth of the high deposition rate film increases the concentration of oxygen vacancies in the film; In addition, the broadening of the diffraction peaks in the XRD spectrum and the results of TEM selected area electron diffraction indicate that there is neutral $\mathrm{SnO}_{2}$ segregation at the grain boundaries of the sample $R_{1}$ and $R_{2}$ films, which reduce thedoping concentration of $\mathrm{Sn}^{4+}$ to $\mathrm{In}^{3+}$ in the lattice. The high deposition rate shows little effect on the doping ratio of $\mathrm{Sn}^{4+}$. Therefore, the carrier concentration of the ITO thin film in the experiment increases with the increasing of deposition rate. In the oxygen flow experiment, the intense (400) peak in the XRD spectrum shows that the carriers of samples $\mathrm{O}_{30}$ and $\mathrm{O}_{40}$ are mainly derived from high-concentration oxygen vacancies formed during the deposition process of low oxygen flow. And because the oxygen flow rate is small during the deposition process, the oxygen ion-assistant energy is relatively low, which is not conducive to the formation of substitutional doping of $\mathrm{Sn}^{4+}$ in the film lattice, The carrier concentrations of $\mathrm{O}_{30}$ and $\mathrm{O}_{40}$ are less than $\mathrm{R}_{3}$ and $\mathrm{R}_{4}$. And for sample $\mathrm{O}_{50}$, the crystal plane diffraction peaks of strong (222) and very weak (400) appearing in the XRD spectrum indicate that the concentration of oxygen vacancies in the film is low, But the increase in oxygen flow increases the auxiliary energy of oxygen ions, making $\mathrm{Sn}^{4+}$ obtain higher energy during the deposition process to enter the crystal lattice to form substitutional doping. Therefore, the carrier concentration in $\mathrm{O}_{50}$ is lower than $\mathrm{O}_{30}, \mathrm{O}_{40}$ and slightly higher than $\mathrm{R}_{2}$. The increase in the carrier concentration of the sample $\mathrm{O}_{60}$ is due to the increasing in the amount of oxygen during the deposition process, which increases the pressure in the vacuum chamber, reduces the mean free path of ion, resulting in the reduction of the assistant energy of oxygen ions, the film atoms are not fully oxidized, the oxygen vacancy concentration in the film slightly rises, and appears as a relative enhancement of the diffraction peak (400) in the XRD spectrum..

The carrier mobility of the ITO film increases first and then decreases with the increasing of deposition rate, from $25.9 \mathrm{~cm}^{2} \mathrm{~V}^{-1} \mathrm{~s}^{-1}$ for $\mathrm{R}_{1}$ to $14.9 \mathrm{~cm}^{2} \mathrm{~V}^{-1} \mathrm{~s}^{-1}$ for $\mathrm{R}_{2}$ and $10.5 \mathrm{~cm}^{2} \mathrm{~V}^{-1} \mathrm{~s}^{-1}$ for $\mathrm{R}_{3}$ and $\mathrm{R}_{4}$. On the other hand, the carrier mobility of the film changes with the amount of oxygen flow rate from $15.1 \mathrm{~cm}^{2} \mathrm{~V}^{-1} \mathrm{~s}^{-1}$ of $\mathrm{O}_{30}$ and $\mathrm{O}_{40}$ rise to $47.8 \mathrm{~cm}^{2} \mathrm{~V}^{-1} \mathrm{~s}^{-1}$ of $\mathrm{O}_{50}$ and then slightly down to 42.6 $\mathrm{cm}^{2} \mathrm{~V}^{-1} \mathrm{~s}^{-1}$ of $\mathrm{O}_{60}$.

The significant decrease in carrier mobility of thin film samples in the experiment can be attributed to the ionized impurity scattering mechanism ${ }^{[12]}$. For samples with higher carrier concentration, $\mathrm{R}_{3}, \mathrm{R}_{4}, \mathrm{O}_{30}$ and $\mathrm{O}_{40}$, high-concentration substitutional doping and the formation of oxygen vacancies cause a large number of ionized impurity centers in the film, and an additional short-range potential will form around it, which will disturb the electromagnetic field in vicinity, hinder carrier transport, and reduce carrier mobility. The ionized impurity scattering mechanism in the transparent conductive semiconductor film can be described by equation:

$$
\mu_{H S}=\frac{4 e}{h}\left(\frac{\pi}{3}\right)^{\frac{1}{3}} N^{-\frac{2}{3}}
$$

Where, $\mathrm{h}$ is Planck constant, $\mathrm{N}$ is the carrier concentration in the film, it can be seen from equation (3) 
that the attenuation of ionized impurity scattering on carrier mobility increases with the increasing of carrier concentration. At the same time, higher doping concentration can also cause lattice distortion, destroy the periodic potential field of electron cloud, and form the static lattice defect scattering. On the contrary, the carrier concentration in sample $\mathrm{R}_{1}, \mathrm{R}_{2}, \mathrm{O}_{50}$ and $\mathrm{O}_{60}$ is relatively lower, and ionized impurity scattering and lattice defect scattering have relatively less effect on carrier mobility.

Since the ITO thin film is n-type semiconductor material which carriers are free electrons, and the mean free path of electrons in the film can be calculated using the heavily doped semiconductor electron cloud $\operatorname{model}^{[13]:}$

$$
L=\left(3 \pi^{2}\right)^{\frac{1}{3}} \cdot h \cdot e^{-2} \cdot \sigma \cdot N^{\frac{2}{3}}
$$

Where, $\mathrm{h}$ is the Plank constant, e is electron charge, $\sigma$ is conductivity (reciprocal of resistivity), $\mathrm{N}$ is the electron concentration in the film. The calculation results are listed in Table 1. Previous studies showed that the electron mean free path of heavily doped n-type semiconductor films is usually of the magnitude of 4 $\mathrm{nm}^{[14]}$. In this experiment, the mean free path of electrons in other samples is between $3 \sim 7 \mathrm{~nm}$ excepting in sample $\mathrm{R}_{1}$, which is much smaller than film grain size. Therefore, the grain boundary scattering mechanism contributes less to the carrier mobility. The mean free path of electrons in sample $R_{1}$ is in the same order of magnitude as the grain size, so the grain boundary scattering mechanism has a considerable influence on the carrier mobility of sample $\mathrm{R}_{1}$. On the other hand, $\mathrm{XRD}$ and TEM test results show that neutral $\mathrm{SnO}_{2}$ precipitates at the grain boundaries of the film during the low deposition rate and the oxygen-rich deposition environment makes some neutral oxygen atoms adsorb at the grain boundaries of the film, which cause a certain concentration of neutral impurity scattering centers in the film. According to Erginsoy model ${ }_{[15]}$, the effect of neutral impurity scattering on mobility is proportional to the concentration of neutral impurities, can be expressed by:

$$
\mu_{N}=\frac{m^{*} e^{3}}{20 \hbar^{3} \varepsilon_{0} \varepsilon_{f} N_{n}}
$$

Which, $\hbar$ is the Reduce the Planck constant, $\varepsilon_{0}$ is the permittivity of vacuum, $\varepsilon_{\mathrm{f}}$ is the thin film dielectric constant, $\mathrm{N}_{\mathrm{n}}$ is the neutral impurity concentration. Therefore, compared with sample $R_{2}$, the neutral impurity scattering mechanism in sample $\mathrm{R}_{1}$ is relatively significant, and its carrier mobility is relatively lower.

\subsection{Optical Performance}

Figure 5 (a) and (b) are the transmittance curves of ITO thin films prepared at different deposition rates and oxygen flow rate in the visible spectrum $(400 \sim 800 \mathrm{~nm})$ (including the influence of sapphire substrate and its average transmittance in the visible spectrum is $87 \%$ ). As can be seen from the figure, the main variation of visible spectrum transmittance of ITO film is to decrease with the increasing of deposition rate, and to increase with the increasing of oxygen flow rate.
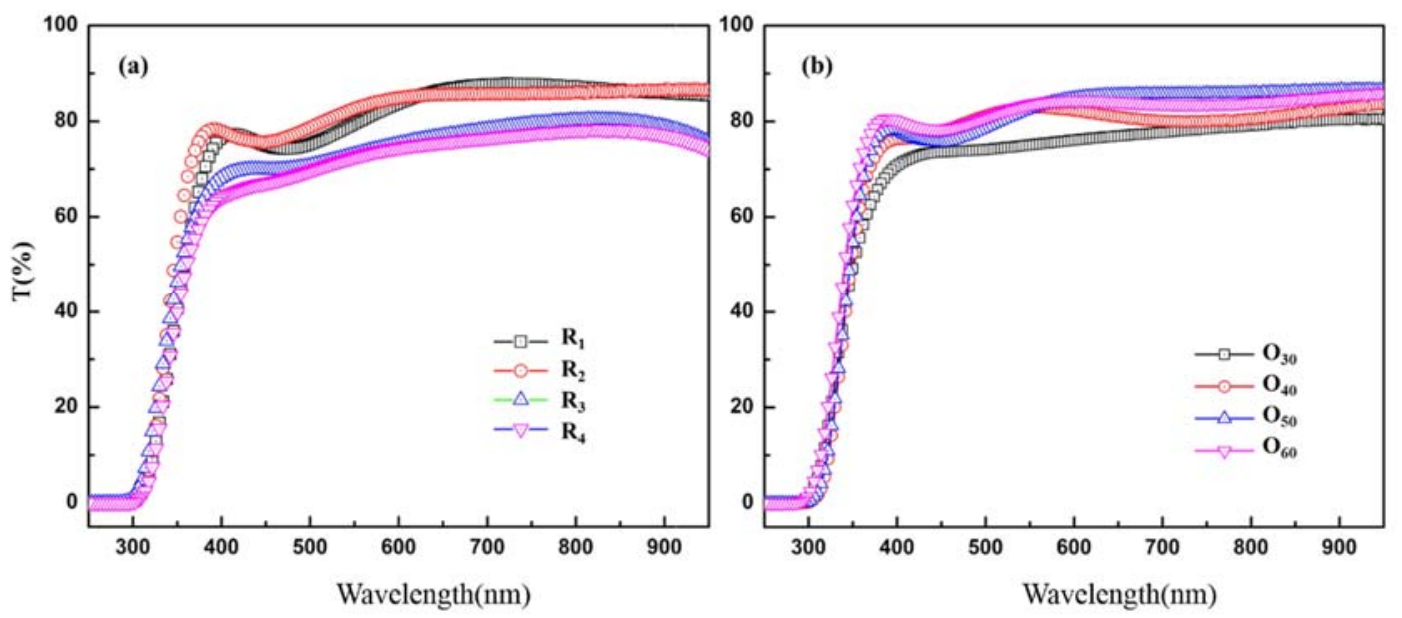

Fig.5 Transmission of as-deposited ITO thin films within visible spectrum

The energy attenuation of the ITO thin film to the light can be attributed to the scattering of photons by absorption and scattering centers. In this paper, high deposition rate and low oxygen flow rate made the films growing in a relatively oxygen-deficient environment, and nonstoichiometric In and Sn low-valent oxides formed in the film which have strong absorption of visible light result in the decreasing of visible spectrum transmittance. At the same time, the ITO thin films prepared under the conditions of high deposition rate and low oxygen flow rate had more lattice defects and structural defects in the film have a large scattering effect on photons which reduces the transmittance of visible light.

\section{Conclusion}


ITO thin films were deposited on sapphire substrates under different deposition parameters via ion-assistant electron beam evaporation method. XRD test results show that the ITO thin films obtained in the experiment are all polycrystalline, the intensity of (400) plane peak, which correlated to the oxygen vacancy concentration in the film, changed with the variation of deposition conditions; Electrical performance test results show that the resistivity of the film is on the order of $10^{-4} \Omega \cdot \mathrm{cm}$ and the carriers were mainly derived from the oxygen vacancies formed during the film deposition and the substitutional doping of $\mathrm{Sn}^{4+}$ ions to $\mathrm{In}^{3+}$ ions in the crystal lattice. The oxygen vacancy concentration increases with the increasing of deposition rate, and decreases with the increasing of oxygen flow rate within a certain range. When the oxygen flow rate reached $60 \mathrm{sccm}$ during the deposition process, the increase in deposition pressure weakens the assistant energy of the $\mathrm{O}$-ion beam which resulting in insufficient oxidation of the film atoms and a slight increase in the oxygen vacancy concentration. In addition, the TEM-SAED shows that neutral $\mathrm{SnO}^{2}$ impurities are formed in the thin film at low deposition rates, which weakens the substitutional doping of $\mathrm{Sn}^{4+}$ to $\mathrm{In}^{3+}$ in the lattice and reduces the donor concentration. The carrier mobility of ITO thin film is determined by multiple scattering mechanisms. The mobility of the high carrier concentration thin film is mainly affected by the ionized impurity scattering mechanism, and the mobility decreases with the increase of the carrier concentration. However, the concentration of neutral particles in the low deposition rate film is relatively high, and the scattering effect of neutral impurities is intensified. At the same time, compared with large-scale grain film, the grain size is in the same order of magnitude as the mean free path of electron motion and grain boundary scattering has a certain influence on carrier mobility; The decrease in the visible light transmittance of the thin films is mainly due to scattering of visible light photons of absorption of light waves by low-valent In and $\mathrm{Sn}$ oxides formed in an oxygen-deficient deposition environment and the structural defects formed during film deposition.

\section{References}

1. WANG Xiu-juan, SI Jia-le, YANG De-lin, et al. Preparation of ITO Thin Films by RF Magnetron Sputtering at Low Temperature[J]. Journal of Synthetic Crystals, 2015, 44(06): 1516-1522.

2. JIANG Min-hong, LIU Xin-yu. Research on annealing process and properties of Al-doped $\mathrm{ZnO}$ films[J]. Transactions of Materials and Heat Treatment, 2009, 30(2): 53-56.

3. HUANG Jun-yi, FAN Guang-han, ZHANG Yong. Institute of Opto-electronic Materials and Technology[J], Transactions of Materials and Heat Treatment, 2010, 31(7): 25-29.

4. V. Senthilkumar, P. Vickrama*, M.Jayachandran, et al. Structure and optical properties of indium Tin
Oxide (ITO) thin films with different compositions prepared by electron beam evaporation [J]. Vacuum, 2010, 84: 864-869.

5. Kwang-Hyuk Choi, Jihoon Kim, Young-Jin Noh, et al. Ag nanowire-embedded ITO films as a nearinfrared transparent and flexible anode for flexible organic solar cells [J].Solar Energy Materials \& Solar Cells, 2013,110:147-153.

6. Mariya Aleksandrova*, Nikolay Kurtev, Valentin Videkov, et al. Material alternative to ITO for transparent conductive electrode in flexible display and photovoltaic devices [J].Microelectronic Engineering, 2015,145: 112-116.

7. I. Del Villar*, C.R. Zamarreňo, M. Hernaez, et al. Generation of surface plasmon resonance and lossy mode resonance by thermal treatment of ITO thinfilms [J]. Optics \& Laser Technology, 2015,69: 1-7.

8. R.L. Weiher. Electrical properties of single crystal of indium oxide [J].Journal of Applied Physics, 1962, 33: 2834-2840.

9. Akio Suzuki, Tatsuhiko Matsushita, Takanori Aoki, et al. pulsed laser deposition of transparent conducting indium tin oxide films in magnetic field perpendicular to plume $[\mathrm{J}]$. Japanese Journal of Applied Physics,2001,40: L401-L403.

10. Hiromichi ohta, masahiro orita, masahiro hirano. highly electrically conductive indium-tin-oxide thin films epitaxially grown on yttria-stabilized zirconia (100) by pulsed-laser deposition [J]. Applied Physics Letter, 2000,76(19): 2740-2742.

11. D. Mergel, W. Stass, G. Ehl, et al. Oxygen incorporation in thin films of In2O3: Sn prepared by radio frequency sputtering [J].Journal of Applied Physics,2000,88: 2437-2442.

12. V.A. Johnson, K. Lark-Horovitz. Theory of thermoelectric power in semiconductors with applications to germanium[J].Physical Review, 1953,92: 226-232.

13. S.K. Choi, J.I. Lee. Effect of film density on electrical properties of indium thin oxide films deposited by Dc magnetron reactive sputtering [J].Journal of Vacuum Science \& Technology A,2001,19(5): 2043-2047.

14. Timothy J.Coutts, Thomas O.Mason, John D.Perkins, et al. Transparent conducting oxides: status and opportunities in basic research[C]. 195th Meeting of the Electrochemical Society Seattle, U.S.A Washington, 1999: 6.

15. Erginsoy C. Neutral impurity scattering in semiconductor [J].Physics Review Letters, 1950,79: 1013-1014. 